\title{
Prophylactic bilateral salpingectomy as a prevention strategy in women at high-risk of ovarian cancer: mini-review
}

\author{
Tess Schenberg ${ }^{1}$ and Gillian Mitchell ${ }^{2,3}$ * \\ ${ }^{1}$ The Western Health Public Hospital, Melbourne, VIC, Australia \\ 2 Peter MacCallum Cancer Centre, Melbourne, VIC, Australia \\ ${ }^{3}$ Sir Peter MacCallum Cancer Centre Department of Oncology, University of Melbourne, Melbourne, VIC, Australia
}

\section{Edited by:}

Viive Maarika Howell, University of Sydney, Australia

Reviewed by:

Tamara Louise Kalir, Mount Sinai School of Medicine, USA

Janice Kwon, The University of British

Columbia, Canada

*Correspondence:

Gillian Mitchell, Familial Cancer Centre, Peter MacCallum Cancer

Centre, Locked Bag 1, A'Beckett

Street, Melbourne, VIC 8006,

Australia

e-mail: gillian.mitchell@petermac.org
Risk-reducing bilateral salpingo-oophorectomy is a proven strategy to reduce the risk of serous ovarian cancer associated with germline BRCA mutations. It is most effective when performed before natural menopause, but it will render a woman prematurely menopausal. The tubal hypothesis of serous ovarian cancer brings with it the possibility of the alternative surgical approach in younger women comprising of risk-reducing bilateral salpingectomy while conserving their ovaries until nearer the age of natural menopause, when a delayed bilateral oophorectomy can be performed. This article will review the evidence behind the tubal hypothesis of serous ovarian cancer and explore the opportunities for translating this into clinical cancer prevention practice.

Keywords: BRCA, bilateral salpingectomy, ovarian cancer, tubal cancer, bilateral salpingo-oophorectomy, cancer prevention
Women at a high-risk of developing serous ovarian cancer due to their inheritance of a germline mutation in a cancer predisposition gene, such as BRCA1, BRCA2 (1), are strongly advised to have prophylactic surgery to remove their ovaries and fallopian tubes (riskreducing bilateral salpingo-oophorectomy RRBS0) once childbearing is complete $(2,3)$. Screening for ovarian cancer in high-risk populations is not recommended although a recent report suggests a degree of tumor down-staging with a strict adherence to an intensive screening protocol (4). No mortality benefit has been shown for ovarian cancer screening, even with strict adherence to screening protocols, in contrast to a clear mortality reduction with RRBSO in this population (5). Premenopausal BSO also brings a $50 \%$ reduction in breast cancer incidence in this high-risk group (5) reinforcing the recommendation for early RRBSO.

The timing of an RRBSO is crucial as the stakes are high. On one hand there is the risk of death from cancer, but this needs to be balanced by the potential for significant morbidity and occasional mortality as a consequence of the procedure itself. These are often young patients without cancer and if guided to the wrong prophylactic strategy, they could develop invasive and potentially incurable cancer. However, the risks of the procedure itself also need to be considered, including the immediate surgical and anesthetic risks but also the medical and psychological complications of plunging a woman into a premature menopause.

RRBSO can be a morbid procedure, particularly for younger premenopausal women, although the majority report a positive outcome overall (6-8). In the non-high-risk population, a bilateral oophorectomy at a younger age is associated with increased all-cause mortality $(9,10)$, predominantly related to the increased risk of cardiovascular disease (11). Reportedly, there is also an increased risk of Parkinsonism, cognitive impairment or dementia (12-14), and osteoporosis (15). While there are good prospective data to support a short-term improvement in mortality for RRBSO in high-risk women (5), the very long-term effects on morbidity and mortality in this group are unknown (6). Obviously, any option to prevent women experiencing an early menopause is going to be attractive to both clinicians and patients. Since the tubal hypothesis of ovarian cancer was first published in 2007 (16), there has been increasing discussion about a staged approach of initial bilateral salpingectomy (RRBS) once childbearing is complete, followed by a delayed oophorectomy (RRBO) closer to natural menopause (17-19).

Prior to 2001, the hypothesis underlying the pathogenesis of ovarian cancer implicated the ovarian surface epithelium or cortical epithelial inclusions that occur during ovulation, with the different ovarian cancer subtypes due to cellular metaplasia. Once initiated the ovarian cancer would then spread to the fallopian tube and other gynecological organs and the wider pelvic and abdominal cavities. In 2001, reports of a high rate of tubal neoplastic lesions in the RRBSO specimens from high-risk women were published $(20,21)$. In these reports, fallopian tubes of highrisk women were carefully examined and preinvasive cancerous lesions were found leading to other reports with similar findings $(22,23)$ and the unifying hypothesis by Crum et al. suggesting that the fallopian tubes were the site of origin of many serous ovarian cancers (16). These precursor lesions - tubal intraepithelial carcinomas (TICs) - had no correlating precursor lesions within the ovary. When specimens from women with serous ovarian cancers, untested for $B R C A$ mutations, were examined these lesions were also found in at least $40-60 \%$ of cases and the fimbrial end of 
the fallopian tube obliterated in another $20 \%(24,25)$. Further support of the tubal origin hypothesis came from the highly similar cytological features and striking molecular similarities between TICs and invasive high grade ovarian cancers (25). These include identical TP53 mutations, a high proliferation rate, chromosomal instability, and gene expression profiles, which all support a clonal origin (26-28).

A refinement to the tubal hypothesis is that the fimbrial ends of the tubes appear to be most vulnerable to malignant transformation, which may explain why tubal ligation provides some ovarian cancer protection in BRCA mutation carriers as well as women in the general population (29). In 2006, researchers from Boston described a protocol for sectioning and extensively examining the fimbrial end of the fallopian tubes (SEE-FIM). The fimbria were an area of interest as they are exposed to the peritoneal cavity, are in close proximity to the ovarian surface, merge with the serosal mesothelium, and often contain transitional metaplasia (26). It was found, and subsequently confirmed by others using the same sectioning technique, that the fimbria were the most common place for precancerous and non-invasive malignant precursor lesions within the fallopian tubes $(26,30,31)$. Molecular analyses confirm these observations; within the non-neoplastic mucosa of the distal tubes was a benign precursor entity consisting of foci of strong p53 immunostaining (indicative of a TP53 mutation), subsequently termed the "p53 signature." The p53 signature was equally common in non-neoplastic tubes from $B R C A$ mutation carriers and controls, but was observed more frequently and was multifocal in fallopian tubes that also contained TIC. Like the prior studies of TIC, p53 signatures predominated in the fimbriae $(23,30)$. However, despite the predilection for the fimbriae, approximately one-third of TIC lesions have been observed elsewhere in the tube reinforcing the need for total removal of the tube for risk-reducing purposes (32).

From these data a plausible biological model for the pathogenesis of what might be a large proportion of high grade serous ovarian carcinoma has emerged. The hypothesized pathway begins with areas of non-neoplastic distal fallopian tubes developing TP53 mutations. The hypothesis then suggests that this leads to a noninvasive malignancy that eventually dedifferentiates into invasive malignancy that subsequently implants into the ovary. A prospective review of RRBSO specimens from women at high-risk of ovarian cancer due to their family history or known BRCA mutations is supportive of this hypothesis. Of 360 RRBSO specimens reviewed, four invasive malignancies and four TICs were identified - all of which were associated with the tubal epithelium (33).

Clearly this is a compelling theory with a persuasive, although still incomplete, body of evidence behind it and could provide a rationale for risk-reducing bilateral salpingectomy (RRBS). However, it may not be the only route for the pathogenesis of ovarian cancer because the timeframe of the pathogenic process and the point of transfer of malignant or potentially malignant tubal cells to the ovary are not known. It is clear that even when utilizing the FEE-SIM protocol to examine RRBSO specimens there are still ovarian cancers identified that are not associated with any obvious fallopian tube malignancy/pre-malignant lesion. It may be that the tubal primary is too small to be found and/or that another, intra-ovarian, pathway also leads to ovarian cancer and/or that the tubal cells can be transferred to the ovary at a much earlier time point. For example, it may be that during ovulation cortical inclusion cysts are formed incorporating normal tubal epithelial cells (endosalpingiosis), which can then cause carcinoma with an underlying molecular signature consistent with the fallopian tubes (25). If any of these additional theories are correct then high-risk women may be done a serious disservice by neglecting to perform an oophorectomy with salpingectomy.

The evidence supporting the tubal hypothesis of ovarian cancer has already led to calls for bilateral salpingectomy to be added to hysterectomies performed for benign reasons in women at average population risk of ovarian cancer. This was first proposed in 2009 by Salvador et al (34) and has led to a $20 \times$ increase in salpingectomy with hysterectomy in Canada (25) although there are still barriers to its routine implementation (35-37). Adding salpingectomy to hysterectomy does not appear to have any immediate increase in complications (38). Additional proposals to perform salpingectomy rather than tubal ligation for women seeking permanent contraception have also been proposed (25).

While the tubal hypothesis is an intriguing one and can be easily integrated into routine care of women at population risk of ovarian cancer requiring hysterectomy or contraception, is the risk:benefit balance tipped in favor of a staged RRBS followed by risk-reducing bilateral oophorectomy (RRBO) at a later date in younger women at high-risk of ovarian cancer? A Canadian group has developed a Markov Monte Carlo simulation model to compare three strategies for risk reduction in women with $B R C A$ mutations: (1) RRBSO; (2) RRBS; and (3) RRBS with delayed RRBO (18). The model estimated the number of future breast and ovarian cancers and cardiovascular deaths attributed to premature menopause with each strategy. RRBSO was the most effective risk-reducing strategy but RRBS with delayed RRBO was still cost effective for those women unwilling to have a RRBSO.

Despite the evidence presented above, unfortunately the point has not yet been reached where the tubal hypothesis of ovarian cancer can be reliably used to guide decision-making around prophylactic surgery in high-risk women (39). To safely change current recommendations, we need prospective evidence that the strategy of a staged approach is not inferior to upfront RRBSO. A randomized controlled trial comparing these strategies is unfortunately not feasible. The difficulties inherent in this approach are obvious, recruiting from a highly selected group of patients will take an international effort over many years in order to give sufficient statistical power to detect a state of non-inferiority, but there is also the ethical dilemma for clinicians offering randomization to an untested procedure against one, which has proven mortality benefits in a young population - would enough clinicians be in clinical equipoise in order to recruit sufficient numbers of participants? A prospective cohort study following high-risk women selecting RRBS over RRBSO (risk-reducing bilateral salpingooophorectomy) is a more practicable approach to answer the question but would still require a large population to give a statistically significant result. It is unlikely that a single international cohort study will be proposed and funded to answer this question but there are a number of prospective cohort studies in BRCA mutation carriers across the world that could provide the necessary outcome data in the future provided the required data can be 
collected systematically. Furthermore, many familial cancer clinics follow up mutation carriers and would also be in a position to contribute prospective outcome data in the future. Provided that all of these groups can be brought together to pool data, an answer may be forthcoming.

So, what to advise a young BRCA mutation carrier who has completed her family while still in her 30 s, or is in her 40 s and declines RRBSO? Careful counseling is necessary to ensure that she is fully informed about the range of surgical prevention options, explaining the risks, and benefits, of all surgical approaches. It is necessary to emphasize the known mortality and breast cancer risk reduction benefits of RRBSO, and ensure that she is aware of the range of strategies to manage any sequelae arising from a premature surgical menopause. The advantage of the alternative of a staged procedure starting with bilateral salpingectomy then a bilateral oophorectomy at or approaching the age of natural menopause is that it avoids morbidity of premature menopause but this comes at the cost of uncertain impact on overall mortality, ovarian cancer-specific mortality and abrogation, or complete loss of breast cancer risk reduction. The Markov model (18) concluding that RRBS with delayed RRBO salpingectomy followed by delayed oophorectomy yields the highest quality-adjusted life expectancy (18) is intriguing, however, it is essential for a fully informed decision that it is made clear to the high-risk woman that no prospective data yet exists on the efficacy of bilateral salpingectomy in reducing mortality in high-risk women. However, in the end, it is a woman's decision based on her own preferences and life experiences and it is the role of her medical team to support her in her choices in order to maximize their benefit and minimize their risk. Some prophylactic surgery in the form of bilateral salpingectomy is probably better than no surgery in this high-risk population.

\section{AUTHOR CONTRIBUTIONS}

Tess Schenberg and Gillian Mitchell, article concept, article drafting, and final approval of manuscript.

\section{REFERENCES}

1. Mavaddat N, Peock S, Frost D, Ellis S, Platte R, Fineberg E, et al. Cancer risks for BRCA1 and BRCA2 mutation carriers: results from prospective analysis of EMBRACE. J Natl Cancer Inst (2013) 105(11):812-22. doi:10.1093/jnci/djt095

2. National Comprehensive Cancer Network (NCCN). Genetic/High-Risk familial Assessment: Breast and Ovarian 2013. (Vol. 4). (2013). [Available from: http://www.nccn.org/professionals/physician_gls/pdf/genetics_screening.pdf

3. National Institute of Health and Care Excellence (NICE). Familial Breast Cancer: Classification and Care of People at Risk of Familial Breast Cancer and Management of Breast Cancer and Related Risks in People with a Family History of Breast Cancer. London: National Institute for Health and Care Excellence (2013). [Available from: http://www.nice.org.uk/nicemedia/live/14188/64204/ 64204.pdf

4. Rosenthal AN, Fraser L, Manchanda R, Badman P, Philpott S, Mozersky J, et al. Results of annual screening in phase I of the United Kingdom familial ovarian cancer screening study highlight the need for strict adherence to screening schedule. J Clin Oncol (2013) 31(1):49-57. doi:10.1200/JCO.2011.39.7638

5. Domchek SM, Friebel TM, Singer CF, Evans DG, Lynch HT, Isaacs C, et al. Association of risk-reducing surgery in BRCA1 or BRCA2 mutation carriers with cancer risk and mortality. JAMA (2010) 304(9):967-75. doi:10.1001/jama.2010. 1237

6. Finch A, Narod SA. Quality of life and health status after prophylactic salpingooophorectomy in women who carry a BRCA mutation: A review. Maturitas (2011) 70(3):261-5. doi:10.1016/j.maturitas.2011.08.001
7. Finch A, Metcalfe KA, Chiang J, Elit L, McLaughlin J, Springate C, et al. The impact of prophylactic salpingo-oophorectomy on quality of life and psychological distress in women with a BRCA mutation. Psychooncology (2013) 22(1):212-9. doi:10.1002/pon.2041

8. Pezaro C, James P, McKinley J, Shanahan M, Young MA, Mitchell G. The consequences of risk reducing salpingo-oophorectomy: the case for a coordinated approach to long-term follow up post surgical menopause. Fam Cancer (2012) 11(3):403-10. doi:10.1007/s10689-012-9527-5

9. Rocca WA, Grossardt BR, de Andrade M, Malkasian GD, Melton LJ. Survival patterns after oophorectomy in premenopausal women: a population-based cohort study. Lancet Oncol (2006) 7(10):821-8. doi:10.1016/S1470-2045(06) 70869-5

10. Parker WH, Feskanich D, Broder MS, Chang E, Shoupe D, Farquhar CM, et al. Long-term mortality associated with oophorectomy compared with ovarian conservation in the nurses' health study. Obstet Gynecol (2013) 121(4):709-16. doi:10.1097/AOG.0b013e3182864350

11. Ingelsson E, Lundholm C, Johansson AL, Altman D. Hysterectomy and risk of cardiovascular disease: a population-based cohort study. Eur Heart J (2011) 32(6):745-50. doi:10.1093/eurheartj/ehq477

12. Rocca WA, Bower JH, Maraganore DM, Ahlskog JE, Grossardt BR, de Andrade M, et al. Increased risk of cognitive impairment or dementia in women who underwent oophorectomy before menopause. Neurology (2007) 69(11):1074-83. doi:10.1212/01.wnl.0000276984.19542.e6

13. Rocca WA, Bower JH, Maraganore DM, Ahlskog JE, Grossardt BR, de Andrade $\mathrm{M}$, et al. Increased risk of parkinsonism in women who underwent oophorectomy before menopause. Neurology (2008) 70(3):200-9. doi:10.1212/01.wnl. 0000280573.30975.6a

14. Rocca WA, Grossardt BR, Maraganore DM. The long-term effects of oophorectomy on cognitive and motor aging are age dependent. Neurodegener Dis (2008) 5(3-4):257-60. doi:10.1159/000113718

15. WHO Scientific Group. Prevention and Management of Osteoporosis. WHO Technical Report Series No. 921. Geneva: Word Health Organization (2003).

16. Kindelberger DW, Lee Y, Miron A, Hirsch MS, Feltmate C, Medeiros F, et al. Intraepithelial carcinoma of the fimbria and pelvic serous carcinoma: Evidence for a causal relationship. Am J Surg Pathol (2007) 31(2):161-9. doi:10.1097/01. pas.0000213335.40358.47

17. Anderson CK, Wallace S, Guiahi M, Sheeder J, Behbakht K, Spillman MA. Risk-reducing salpingectomy as preventative strategy for pelvic serous cancer. Int J Gynecol Cancer (2013) 23(3):417-21. doi:10.1097/IGC.0b013e3182849dba

18. Kwon JS, Tinker A, Pansegrau G, McAlpine J, Housty M, McCullum M, et al. Prophylactic salpingectomy and delayed oophorectomy as an alternative for BRCA mutation carriers. Obstet Gynecol (2013) 121(1):14-24. doi:10.1097/ AOG.0b013e3182783c2f

19. Leblanc E, Narducci F, Farre I, Peyrat JP, Taieb S, Adenis C, et al. Radical fimbriectomy: a reasonable temporary risk-reducing surgery for selected women with a germ line mutation of BRCA 1 or 2 genes? Rationale and preliminary development. Gynecol Oncol (2011) 121(3):472-6. doi:10.1016/j.ygyno. 2011.02.012

20. Piek JM, van Diest PJ, Zweemer RP, Jansen JW, Poort-Keesom RJ, Menko $\mathrm{FH}$, et al. Dysplastic changes in prophylactically removed Fallopian tubes of women predisposed to developing ovarian cancer. J Pathol (2001) 195(4):451-6. doi:10.1002/path.1000

21. Colgan TJ, Murphy J, Cole DE, Narod S, Rosen B. Occult carcinoma in prophylactic oophorectomy specimens: prevalence and association with BRCA germline mutation status. Am J Surg Pathol (2001) 25(10):1283-9. doi:10.1097/00000478200110000-00009

22. Crum CP, Drapkin R, Kindelberger D, Medeiros F, Miron A, Lee Y. Lessons from BRCA: the tubal fimbria emerges as an origin for pelvic serous cancer. Clin Med Res (2007) 5(1):35-44. doi:10.3121/cmr.2007.702

23. Folkins AK, Jarboe EA, Saleemuddin A, Lee Y, Callahan MJ, Drapkin R, et al. A candidate precursor to pelvic serous cancer ( $\mathrm{p} 53$ signature) and its prevalence in ovaries and fallopian tubes from women with BRCA mutations. Gynecol Oncol (2008) 109(2):168-73. doi:10.1016/j.ygyno.2008.01.012

24. Przybycin CG, Kurman RJ, Ronnett BM, Shih IM, Vang R. Are all pelvic (nonuterine) serous carcinomas of tubal origin? Am J Surg Pathol (2010) 34(10):1407-16. doi:10.1097/PAS.0b013e3181ef7b16

25. Gilks CB, Miller D. Opportunistic salpingectomy for women at low risk for development of ovarian carcinoma: the time has come. Gynecol Oncol (2013) 129(3):443-4. doi:10.1016/j.ygyno.2013.04.021 
26. Medeiros F, Muto MG, Lee Y, Elvin JA, Callahan MJ, Feltmate C, et al. The tubal fimbria is a preferred site for early adenocarcinoma in women with familial ovarian cancer syndrome. Am J Surg Pathol (2006) 30(2):230-6. doi:10.1097/01.pas.0000180854.28831.77

27. Salvador S, Rempel A, Soslow RA, Gilks B, Huntsman D, Miller D. Chromosomal instability in fallopian tube precursor lesions of serous carcinoma and frequent monoclonality of synchronous ovarian and fallopian tube mucosal serous carcinoma. Gynecol Oncol (2008) 110(3):408-17. doi:10.1016/j.ygyno. 2008.05.010

28. O'Shannessy DJ, Jackson SM, Twine NC, Hoffman BE, Dezso Z, Agoulnik SI, et al. Gene expression analyses support fallopian tube epithelium as the cell of origin of epithelial ovarian cancer. Int J Mol Sci (2013) 14(7):13687-703. doi:10.3390/ijms140713687

29. Antoniou AC, Rookus M, Andrieu N, Brohet R, Chang-Claude J, Peock S, et al. Reproductive and hormonal factors, and ovarian cancer risk for BRCA1 and BRCA2 mutation carriers: results from the International BRCA1/2 Carrier Cohort Study. Cancer Epidemiol Biomarkers Prev (2009) 18(2):601-10. doi:10.1158/1055-9965.EPI-08-0546

30. Lee Y, Miron A, Drapkin R, Nucci MR, Medeiros F, Saleemuddin A, et al. A candidate precursor to serous carcinoma that originates in the distal fallopian tube. J Pathol (2007) 211(1):26-35. doi:10.1002/path.2091

31. Callahan MJ, Crum CP, Medeiros F, Kindelberger DW, Elvin JA, Garber JE, et al. Primary fallopian tube malignancies in BRCA-positive women undergoing surgery for ovarian ancer risk reduction. J Clin Oncol (2007) 25(25):3985-90. doi:10.1200/JCO.2007.12.2622

32. Mingels MJ, Roelofsen T, van der Laak JA, de Hullu JA, van Ham MA, Massuger LF, et al. Tubal epithelial lesions in salpingo-oophorectomy specimens of BRCA-mutation carriers and controls. Gynecol Oncol (2012) 127(1):88-93. doi:10.1016/j.ygyno.2012.06.015

33. Reitsma W, de Bock GH, Oosterwijk JC, Bart J, Hollema H, Mourits MJ. Support of the 'fallopian tube hypothesis' in a prospective series of riskreducing salpingo-oophorectomy specimens. Eur J Cancer (2013) 49(1):132-41. doi:10.1016/j.ejca.2012.07.021

34. Salvador S, Gilks B, Köbel M, Huntsman D, Rosen B, Miller D. The fallopian tube: primary site of most pelvic high-grade serous carcinomas. Int J Gynecol Cancer (2009) 19(1):58-64. doi:10.1111/IGC.0b013e318199009c
35. Reade CJ, Finlayson S, McAlpine J, Tone AA, Fung-Kee-Fung M, Ferguson SE. Risk-reducing salpingectomy in Canada: a survey of obstetrician-gynaecologists. J Obstet Gynaecol Can (2013) 35(7):627-34.

36. Kamran MW, Vaughan D, Crosby D, Wahab NA, Saadeh FA, Gleeson N. Opportunistic and interventional salpingectomy in women at risk: a strategy for preventing pelvic serous cancer (PSC). Eur J Obstet Gynecol Reprod Biol (2013) 170(1):251-4. doi:10.1016/j.ejogrb.2013.06.030

37. Plusquin C, Fastrez M, Vandromme J, Rozenberg S. Factors affecting 'gynaecologists' decision to perform prophylactic oophorectomy concomitantly with hysterectomy: a Belgian survey. Maturitas (2011) 70(4):391-4. doi:10.1016/j. maturitas.2011.09.007

38. Morelli M, Venturella R, Mocciaro R, Di Cello A, Rania E, Lico D, et al. Prophylactic salpingectomy in premenopausal low-risk women for ovarian cancer: primum non nocere. Gynecol Oncol (2013) 129(3):448-51. doi:10.1016/j.ygyno. 2013.03.023

39. Collins IM, Domchek SM, Huntsman DG, Mitchell G. The tubal hypothesis of ovarian cancer: caution needed. Lancet Oncol (2011) 12(12):1089-91. doi:10.1016/S1470-2045(11)70222-4

Conflict of Interest Statement: The authors declare that the research was conducted in the absence of any commercial or financial relationships that could be construed as a potential conflict of interest.

Received: 30 September 2013; accepted: 26 January 2014; published online: 10 February 2014.

Citation: Schenberg T and Mitchell G (2014) Prophylactic bilateral salpingectomy as a prevention strategy in women at high-risk of ovarian cancer: a mini-review. Front. Oncol. 4:21. doi: 10.3389/fonc.2014.00021

This article was submitted to Women's Cancer, a section of the journal Frontiers in Oncology.

Copyright (C) 2014 Schenberg and Mitchell. This is an open-access article distributed under the terms of the Creative Commons Attribution License (CC BY). The use, distribution or reproduction in other forums is permitted, provided the original author(s) or licensor are credited and that the original publication in this journal is cited, in accordance with accepted academic practice. No use, distribution or reproduction is permitted which does not comply with these terms. 